\title{
Fecal Calprotectin in Clinical Practice
}

\section{A Noninvasive Screening Tool for Patients With Chronic Diarrhea}

\author{
Anna Licata, MD,* Claudia Randazzo, MD,* Maria Cappello, MD,* Vincenza Calvaruso, MD,* \\ Giuseppe Butera, MD, * Ada M. Florena, MD, $\dagger$ Sergio Peralta, MD, ${ }^{*}$ Calogero Cammà, MD,* \\ and Antonio Craxi, MD*
}

\begin{abstract}
Background: Surrogate markers of colorectal inflammation are increasingly being recognized as important in differentiating organic from functional intestinal disorders. Fecal calprotectin (FC) can be easily measured in the stool, being released by leukocytes in inflammatory conditions.
\end{abstract}

Aim: We evaluated FC as an index of inflammation in consecutive outpatients referred for colonoscopy for chronic, nonbloody diarrhea.

Methods: Stool specimens of 346 outpatients with chronic, nonbloody diarrhea, referred for colonoscopy, were measured for FC levels. The proportion of patients correctly diagnosed with the test and the relationship with endoscopic and histologic findings were measured.

Results: Abnormal endoscopic findings were detected in 104 patients $(30.1 \%)$. Histologic findings included 142 patients $(41.0 \%)$ with inflammation and $204(59.0 \%)$ without inflammation. Fecal excretion of calprotectin significantly correlated with the finding of inflammation at endoscopy and histology $(P<0.0001)$. When $150 \mathrm{mcg} / \mathrm{g}$ of stool was used as the upper reference limit, FC showed $75.4 \%$ sensitivity and $88.3 \%$ specificity, with $81.7 \%$ positive and $83.7 \%$ negative predictive values for histologic inflammation.

Conclusions: In outpatients referred for colonoscopy a measurement of FC is accurate to identify those with histologic inflammation. Assay of FC may be a reliable and noninvasive screening tool to identify inflammatory causes of chronic, nonbloody diarrhea.

Key Words: fecal calprotectin, chronic diarrhea, histologic inflammation, screening tool

( Clin Gastroenterol 2012;46:504-508)

$\mathrm{D}$ espite expert-based diagnostic criteria, it is still difficult in routine clinical practice to make a differential diagnosis between organic [ie, infections, inflammatory bowel disease (IBD), or neoplasm] and functional intestinal pathology [eg, irritable bowel syndrome (IBS)]..$^{1}$ In fact, only about one third of all patients who present with chronic diarrhea have a firm diagnosis based upon their initial history and physical examination. ${ }^{2}$ Certain signs and

Received for publication July 13, 2011; accepted December 29, 2011. From the *Sezione e U.O.C. di Gastroenterologia, Di.Bi.M.I.S.; and †Sezione di Anatomia Patologica, Università di Palermo, Palermo, Italy.

The authors declare that they have nothing to disclose.

Reprints: Anna Licata, MD, Sezione di Gastroenterologia \& Epatologia, Dipartimento Biomedico di Medicina Interna e Specialistica, Università di Palermo, Piazza Delle Cliniche 2, 90127, Palermo, Italy (e-mail: annalisalicata@yahoo.com).

Copyright (C) 2012 by Lippincott Williams \& Wilkins symptoms associated with chronic diarrhea, such as rectal bleeding, fever, and leukocytosis, are suggestive of inflammatory disorders and, in such cases, stool triage would not be a necessary component of the diagnostic algorithm. For the remaining majority of chronic diarrhea patients the ensuing clinical evaluation can be prolonged, invasive, and resource intensive. ${ }^{3}$

Simple screening tests may be useful for discriminating the presence or absence of organic pathology, and could help direct the diagnostic work-up in a timelier and costeffective manner to avoid inherent risks associated with diagnostic procedures. ${ }^{4}$ Routine blood tests that use biomarkers of inflammation are neither specific nor sensitive enough because they are indirect measures of inflammation. The fecal occult-blood test has a low sensitivity for a diagnosis of colon cancer, especially at an early stage. ${ }^{5}$ In the case of bowel disease, the level of inflammation biomarkers in the stool would appear to be the ideal test for mucosal involvement. ${ }^{6}$

In the past few years, several leukocyte-derived proteins excreted in stools have been proposed as markers of intestinal inflammation. ${ }^{7}$ Fecal calprotectin (FC) is a $36.5 \mathrm{kDa}$, nonglycosylated calcium and zinc binding protein with antimicrobic, immunomodulatory, and antiproliferative properties. It is present in mucous membrane squamous epithelium, but not in the normal intestinal mucosa, and accounts for about $60 \%$ of total (cytosol soluble) proteins in neutrophils granulocytes. ${ }^{8}$ The mucosal barrier is altered in intestinal inflammation, allowing white cells to cross the intestinal wall. Neutrophil determination in stool is inefficient because its brief lifetime makes it mandatory that the sample should be examined within a few hours of its collection. ${ }^{9}$ FC is resistant to bacterial proteolytic degradation during passage through the gastrointestinal tract, and shows high stability in feces $(>7 d)$ at different temperatures, and can be easily measured, ${ }^{10}$ making it a surrogate marker of neutrophils influx into the bowel lumen. As a result, it has been suggested that FC could be considered a simple, noninvasive, and reliable screening method for detecting intestinal inflammation.

Aim of our study was to assess the diagnostic performance of FC as a stool-screening biomarker for organic intestinal disease. Therefore, we assessed FC levels in consecutive adult outpatients with unexplained chronic, nonbloody diarrhea referred for colonoscopy.

\section{MATERIALS AND METHODS}

\section{Patients}

We prospectively studied adult (age of at least $18 \mathrm{y}$ ) consecutive patients referred to evaluate chronic ( $\geq 4 \mathrm{wk}$ ), 
nonbloody diarrhea of unknown origin at our gastroenterology outpatient department (Gastroenterology and Hepatology Unit, University of Palermo) between March 2004 and May 2009.

Exclusion criteria were overt gastrointestinal bleeding, known colorectal or gastric neoplasia, familial adenomatous polyposis and hereditary nonpolyposis colorectal cancer syndrome, history of colonic surgery, recent respiratory or urinary tract infection, acute infectious disease, pregnancy and alcohol abuse (consumption $>30 \mathrm{~g} / \mathrm{d}$ in men and $>20 \mathrm{~g} / \mathrm{d}$ in women), intake of nonsteroidal anti-inflammatory drugs, aspirin, and anticoagulants within the previous month. None of our patients have had a previous diagnosis of colorectal disease.

A full medical history, including current smoking habits, and common use of medications, was obtained from all patients enrolled in the study, and a physical examination was performed. In addition, venous blood samples were taken from all patients for complete blood count and biochemical analysis, including erythrocyte sedimentation rate (ESR), C-reactive protein, serum electrolytes, thyroid hormones, and liver and renal function tests. Stool cultures and search for ova and parasites were done. All patients underwent colonoscopy with biopsies. All patients gave the informed consent for FC evaluation assay and supplied a single stool sample before the bowel preparation for colonoscopy. Small bowel enteroclysis and upper gastrointestinal endoscopy with distal duodenal biopsies were done in all patients in whom Crohn's disease was histologically diagnosed, to define the extension of the disease. Gastroenterologists were unaware of the FC results throughout the study.

Patients with negative results for all of the examinations described above, and with a clinical history indicative of nonorganic disease, were then considered to be suffering from IBS, according to Rome II criteria. ${ }^{11}$ Organic diseases were diagnosed using generally accepted conventional criteria.

Demographic and clinical characteristics, biochemical data, endoscopic, and histologic findings were recorded in an electronic database.

\section{Stool Collections}

Stool specimens were collected and returned by each study subject in a disposable plastic bucket-like device to avoid toilet water artefacts and to simplify laboratory sampling. Upon receipt, stool samples were aliquoted for immediate assay or stored at $-20^{\circ} \mathrm{C}$ until assay.

\section{FC Assay}

Fecal assays were performed at a single laboratory by experienced laboratory technicians, without prior knowledge of the clinical diagnoses or details of the patients' clinical histories. Calprotectin concentrations were determined using the commercially available enzyme-linked immunosorbent assay test (Calprest, Eurospital SpA, Trieste, Italy), a quantitative enzyme-linked immunoassay that uses polyclonal antibodies against calprotectin. Aliquots of approximately $100 \mathrm{mg}$ feces (range, 40 to $120 \mathrm{mg}$ ) were homogenized for $25 \pm 5$ minutes with extraction buffer. After homogenate $(1 \mathrm{~mL})$ centrifugation for $20 \mathrm{mi}$ nutes at $10.000 \mathrm{rpm}$, the supernatant $(0.5 \mathrm{~mL})$ was diluted 1:50. A $100 \mu \mathrm{L}$ sample was added in duplicate to each well, which had been previously coated with antibody. Standards contained calprotectin at known concentrations. After
$45 \pm 5$ minutes of incubation at room temperature, washing was followed by the addition of $100 \mu \mathrm{L}$ of conjugate and, after adding $100 \mu \mathrm{L}$ substrate and incubation at room temperature for 30 minutes in a dark place, the optical density values were read at $405 \mathrm{~nm}$. Results were recorded as a continuous variable (in $\mathrm{mcg} / \mathrm{g}$ of stool) based on preset threshold values supplied by the manufacturer $(<250 \mathrm{mcg} / \mathrm{g}$ of stool = negative, $\geq 250 \mathrm{mcg} / \mathrm{g}$ of stool $=$ positive $)$.

\section{Endoscopy}

Colonoscopies were performed by experienced staff gastroenterologists who were unaware of the FC results. Cecal intubation, coupled with $>90 \%$ mucosal surface visualization, constituted a complete examination. Mucosal abnormalities were recorded by anatomic subsite and biopsies were obtained from the terminal ileum, right colon, left colon, and rectum. The following endoscopic diagnoses were considered: normal endoscopic signs, findings of IBD, diverticular disease with or without peridiverticular inflammation, polyps, and ischemic colitis.

\section{Histologic Examination}

Histopathologic evaluations of colorectal inflammation were made by experienced gastrointestinal histopathologists, who were blinded to the FC results. Histologic inflammation was defined by histologic standard criteria and subtyped as: Crohn's disease, ulcerative colitis, microscopic colitis. Colitis was defined as nonspecific when the pathologist was not able to formulate a diagnosis for lack of clinical data or insufficient information. To correlate FC levels and histologic findings, colorectal inflammation was graded as 0 to $2(0=$ normal mucosa, $1=$ mild inflammation with mild/no, glandular distortion, and $2=$ severe inflammation, with glandular distortion).

\section{Statistical Analysis}

Continuous variables were summarized as median and range, and categorical variables as frequency and percentage. Data were previously examined by a normality test. A $P$ value of $<0.05$ was considered statistically significant.

To predict colorectal inflammation at histology, a backward logistic regression was fitted to the data. The patients were grouped according to the histopathologic findings; a dichotomous variable was assigned to each patient that was a value of 1 for patients with colorectal inflammation and 0 for patients without. Multiple linear regression analysis was done to identify independent predictors of FC levels as continuous dependent variables in all groups of patients.

Box plots were used to provide an immediate graphical evaluation of FC values by the endoscopic and histologic findings. Correlation between the high level of FC and the presence of lesions (endoscopic and histologic) was estimated by the Spearman correlation rank coefficient.

Parameters of FC reliability, including sensitivity, specificity, positive and negative predictive values were calculated by construction of a receiver operating characteristic (ROC) curve. Area under the receiver operating characteristic curve for histologic inflammation versus no inflammation was estimated: 1.0 is characteristic of an ideal test, whereas 0.5 indicates a test of no diagnostic value.

Statistical data analysis was performed by using the Statistical Package for Social Science version 13.0 for Windows. 


\section{RESULTS}

A total of 382 outpatients were interviewed. Of these, $36(9.4 \%)$ were deemed ineligible based on 1 or more exclusion criteria. The remaining $346(90.6 \%)$ patients were included in the study.

In detail, endoscopy was normal in 242 patients $(69.9 \%)$; colorectal polyps were found in $10(2.9 \%)$, IBD in $82(23.7 \%)$, uncomplicated diverticula in $11(3.2 \%)$, and ischemic colitis in 1 patient $(0.3 \%)$. Median levels of FC for the above-specified 5 diagnostic groups are shown in Figure 1 . When compared with patients with normal endoscopy, median FC levels were significantly higher in all the other groups $(P<0.0001$, Spearman correlation rank test $)$.

Histologic findings included 142 patients $(41.0 \%)$ with inflammation and $204(59.0 \%)$ without it. Median levels of FC the above 2 groups are shown in Figure 2. Median FC levels were significantly higher in patients with inflammatory conditions $(P<0.0001$, Spearman correlation rank test) compared with those normal histologic findings.

The main final diagnosis among the patients with histologic inflammation was IBD in 82 (56 Crohn's disease and 26 ulcerative colitis). Miscellaneous conditions were found in 17 (microscopic colitis $=6$; diverticular disease $=4$; polyps $=6$; ischemic colitis $=1$ ). In 22 patients, the pathology findings reported a diagnosis of nonspecific colitis. The remaining 21 patients had a clinical diagnosis of IBS based on the history (Roma criteria) and on the absence of any red flags (screening blood and stool structural colonic evaluation), despite their mild lymphocytic infiltration. The final diagnosis for the group of patients without histologic inflammation was IBS for 197 subjects and diverticular disease in 7 ; in addition we found 4 colonic polyps on colonoscopy.

Demographic and clinical data are provided according to the presence of histologic inflammation (Table 1). Sex distribution $(P=0.014)$ differed among the groups, with a predominance of females without inflammation. Median ESR $(P<0.0001)$ and FC $(P<0.001)$ values were significantly higher in patients with inflammation.

Associations between demographic, laboratory, and histologic data according to FC levels are summarized in Table 2. Age distribution was similar among study

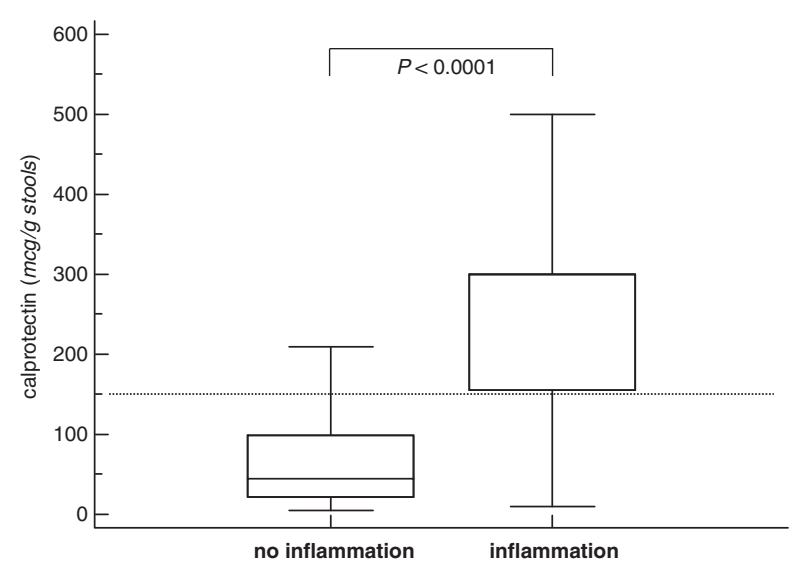

FIGURE 1. Median fecal calprotectin levels according to endoscopic diagnosis. Box and whisker plots showing fecal calprotectin levels ( $\mathrm{mcg} / \mathrm{g}$ of stool) in patients stratified according the endoscopic diagnoses.

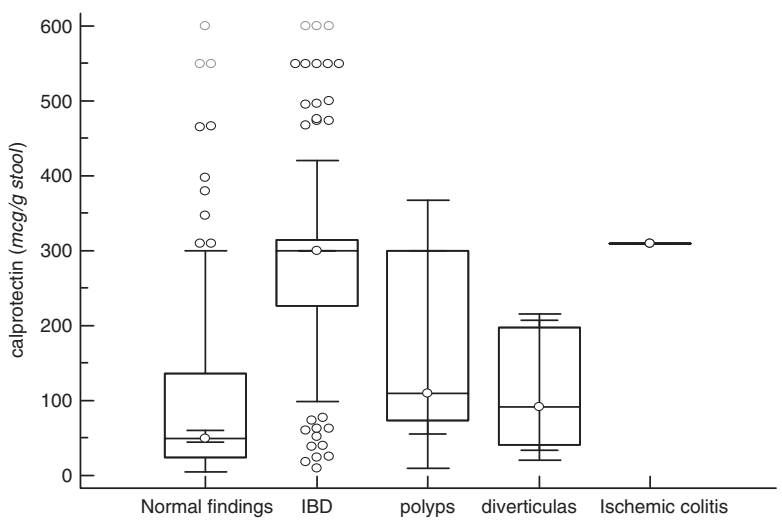

FIGURE 2. Fecal calprotectin distribution according to histologic inflammation. Box and whisker plots showing fecal calprotectin levels $(\mathrm{mcg} / \mathrm{g}$ of stool) in patients stratified in those with and without histologic inflammation. The horizontal line represents the reference value of $150 \mathrm{mcg} / \mathrm{g}$ of stool. $P$ by the Spearman correlation rank coefficient. IBD indicates inflammatory bowel disease.

participants, though sex distributions differed $(P=0.02)$, with females predominance.

Based on a multivariate linear logistic regression model, the presence of histologic inflammation $(P<0.0001$; OR 6.54; 95\% CI, 3.30-12.96) and higher ESR values $(P<0.0001$; OR $1.09 ; 95 \%$ CI, 1.06-1.23) were significantly and independently associated with FC levels.

To appraise the best FC value able to recognize histologic inflammation, we constructed an ROC curve and calculated that the optimized cut-off point for equally important sensitivity and specificity was $>150 \mathrm{mcg} / \mathrm{g}$ of stool (Fig. 3). At this cutoff, FC had $75.4 \%$ sensitivity, $88.3 \%$ specificity, $81.7 \%$ positive predictive values, and $83.7 \%$ negative predictive values in predicting inflammation on histology. Based on ROC curve, FC was a sensitive biomarker for histologic inflammation at all specificities $(\mathrm{AUC}=0.9,95 \% \mathrm{CI}, 0.82-0.89)$.

\section{DISCUSSION}

IBS is probably the most frequent cause of chronic diarrhea in adults, affecting $10 \%$ to $20 \%$ of the general population. ${ }^{12}$ Although simple clinical criteria based on medical history and physical examination are suggested for

TABLE 1. Demographic, ,Laboratory and Histologic Features of 346 Consecutive Patients With Chronic Diarrhea According to Histology

\begin{tabular}{lccc}
\hline & \multicolumn{3}{c}{ Histologic Inflammation } \\
\cline { 2 - 4 } Variable & No $(\mathbf{n}=\mathbf{2 0 4})$ & Yes $(\mathbf{n}=\mathbf{1 4 2})$ & $\boldsymbol{P}$ \\
\hline Sex & $74(36.3)$ & $71(50.0)$ & 0.014 \\
$\quad$ Male & $130(63.7)$ & $71(50.0)$ & \\
Female & $38.0(18-87)$ & $41(17-80)$ & 0.07 \\
Age (y) & $6(2-52)$ & $28(2-115)$ & $<0.0001$ \\
ESR & $0.08(0.01-15.3)$ & $0.47(0.01-7.3)$ & 0.08 \\
CRP (n $=109)$ & $45(5-225)$ & $300(10-600)$ & $<0.001$ \\
FC &
\end{tabular}

Data are given as median (range) or as number of cases $(\%)$.

CRP indicates C-reactive protein; ESR, erythrocyte sedimentation rate; FC, fecal calprotectin. 
TABLE 2. Univariate and Multivariate Analysis of Demographic, Laboratory, and Histologic Features Associated With Elevated FC Levels in 346 Consecutive Patients With Chronic Diarrhea

\begin{tabular}{|c|c|c|c|c|}
\hline & \multicolumn{2}{|c|}{$\begin{array}{c}\text { Univariate } \\
\text { Analysis }\end{array}$} & \multicolumn{2}{|c|}{$\begin{array}{c}\text { Multivariate } \\
\text { Analysis }\end{array}$} \\
\hline & SE & $\boldsymbol{P}$ & OR $(95 \%$ CI $)$ & $\boldsymbol{P}$ \\
\hline Sex & 0.61 & 0.02 & $1.71(0.91-3.20)$ & 0.09 \\
\hline Age (y) & 0.018 & 0.26 & - & - \\
\hline $\begin{array}{l}\text { Histologic } \\
\text { inflammation }\end{array}$ & 0.69 & 0.004 & $6.54(3.30-12.96)$ & $<0.0001$ \\
\hline ESR & 0.02 & 0.01 & 1.09 (1.06-1.23) & $<0.0001$ \\
\hline CRP & 0.27 & 0.23 & - & - \\
\hline
\end{tabular}

$\mathrm{CI}$ indicates confidence interval; CRP, C-reactive protein; ESR, erythrocyte sedimentation rate; FC, fecal calprotectin; OR, odds ratio.

a diagnosis of IBS, ${ }^{13}$ a definitive diagnosis is still reached by excluding other diseases, thus following a more or less aggressive investigation plan. ${ }^{14}$

To limit the numerous and invasive investigations, several noninvasive blood tests have been suggested, but their sensitivity and specificity are quite low, and they do not directly reflect the level of colonic inflammation. ${ }^{15,16}$ Several studies have proposed leukocyte-derived protein assay as a means of identifying inflammatory versus functional causes of chronic diarrhea., ${ }^{9,17-19}$

We tested FC as a biomarker of intestinal inflammation because of its diagnostic capability is considered superior to other acute-phase reactant proteins released during inflammation in the gastrointestinal tract. ${ }^{20} \mathrm{Cal}-$ protectin has characteristics that might facilitate its use including existence in a preformed state within white blood cells, resistance to proteolytic enzymes, stability in stool, and ease in measuring. ${ }^{10,21}$ Moreover, FC has been proposed as a comprehensive marker of organic gastrointestinal disorders. ${ }^{22}$

Our data show that among patients referred for colonoscopy, FC levels are sufficiently accurate in identifying

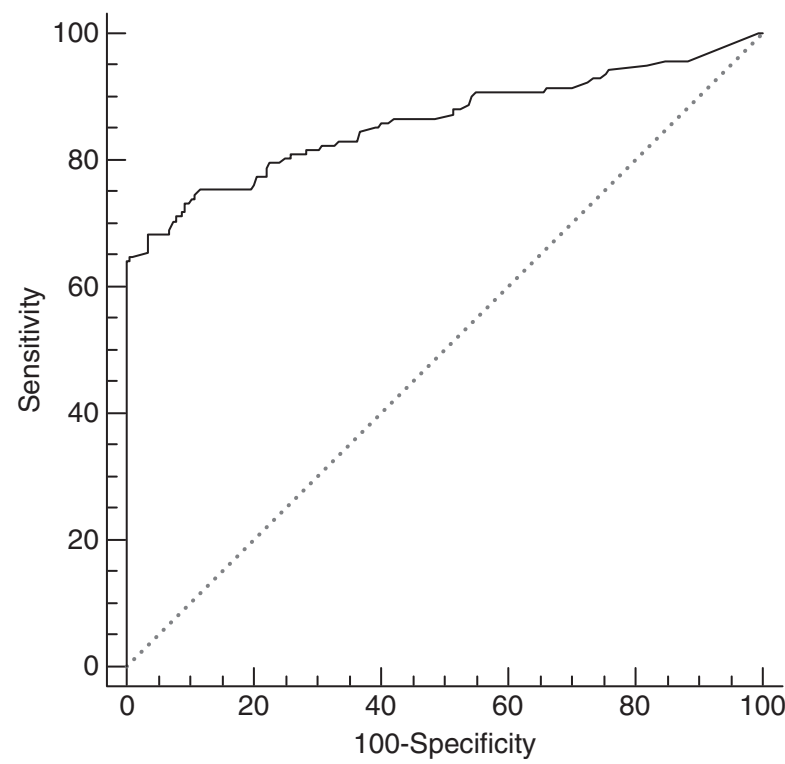

FIGURE 3. Receiver operating characteristic curve for fecal calprotectin in detecting histologic inflammation. those with significant colonic organic disease. In contrast to these findings, Meucci et $\mathrm{al}^{23}$ reported that elevated calprotectin levels were found in as many as $36 \%$ of patients with normal colonoscopy.

In our study, $\mathrm{FC}$ was found to be a biomarker strongly associated with histologic inflammation among colonoscopy referral patients with chronic, nonbloody diarrhea of unknown origin. This finding is biologically intuitive, in as much as leukocytes concentrate within inflamed intestinal mucosa and, unlike erythrocytes, do not require vascular disruption for their luminal release.

To appropriately interpret the fecal assay results, certain features of our patient population are worthy of consideration. All participants had been referred for colonoscopy, which might have led to the selection of an analytic cohort with an unusually high prevalence of organic pathology. However, the proportion of our subjects with inflammatory conditions was not markedly different from that observed in another chronic diarrhea series, ${ }^{2}$ which argues against this possibility. We also used gastrointestinal bleeding as an exclusion criterion, so our findings do not pertain to patients with chronic bloody diarrhea. Nonetheless, this symptom would usually prompt endoscopic evaluation without a need for preliminary stool testing, and does not diminish the potential value of FC screening.

In our study, ROC curve analysis of FC levels showed that a cut-off point of $150 \mathrm{mcg} / \mathrm{g}$ of stool had $75.4 \%$ sensitivity for histologic inflammation, with $83.7 \%$ NPV. These results indicate that the FC assay may be a useful screening tool in the evaluation of patients with chronic, nonbloody diarrhea. The present data are in agreement with previous studies that have shown that FC is a highly sensitive test for mucosal inflammation ${ }^{2,24}$ and a useful marker in discriminating between organic and nonorganic disease. ${ }^{19}$ The reported sensitivity oscillates between $63 \%$ and $95 \%$, whereas specificity has been reported to be between $79 \%$ and $93 \%$. $9,17,18,24$

Using the cut-off point of $150 \mathrm{mcg} / \mathrm{g}$ of stool, about $12 \%$ of the IBS patients had calprotectin levels ranging from 173 to $182 \mathrm{mcg} / \mathrm{g}$ of stool. In such cases, the possibility of day-to-day variations should be kept in mind, as shown by a study in which a marked variability of calprotectin was found on repeated assessments in $64 \%$ of patients without neoplasm or inflammation. ${ }^{25}$ The proposed infective hypothesis could provide a possible explanation for the high fecal calprotectin values in IBS patients. ${ }^{26}$ If true, it is likely that IBS patients will have leukocytes infiltrate in their intestinal wall. Another possibility is the presence of undiagnosed microscopic colitis. The diagnostic value of FC may also be limited in patients with collagenous colitis, as up to $40 \%$ of the patients with active collagenous colitis are reported to have a normal calprotectin excretion. ${ }^{27}$

Our data suggest that resource utilization, especially of expensive and invasive procedures like colonoscopy might be more rationally and cost effectively directed, based on the determination of FC levels. The FC assay may have additional uses beyond its proposed function; by using a combination of clinical factors, such as age, symptoms, and noninvasive fecal markers, a significant proportion of patients with IBS could avoid further invasive investigations, reserving endoscopy only for those with elevated FC concentrations. This strategy is likely to have beneficial resource and cost implications for endoscopy services, as up to $40 \%$ of new gastroenterology referrals are for patients with suspected IBS. ${ }^{28}$ Waiting lists for nonurgent colonoscopy may be many 
months in some centers, and an elevated FC result may indicate that a patient requires urgent endoscopic assessment. Conversely, an FC concentration of $<150 \mathrm{mcg} / \mathrm{g}$ of stool is unlikely to be associated with intestinal inflammation, and investigations can be tailored appropriately.

In conclusion, our results suggest that $\mathrm{FC}$ reflects the inflammatory status of the intestinal mucosa. The simplicity of the methods, as well as the sensitivity, propose that FC may be an ideal routine screening tool for the diagnosis of organic intestinal disease. Our data suggest that resource utilization, especially of expensive and invasive procedures like colonoscopy, might be more rationally and cost effectively directed based on the determination of FC levels.

\section{REFERENCES}

1. Otten CM, Kok L, Witteman BJ, et al. Diagnostic performance of rapid tests for detection of fecal calprotectin and lactoferrin and their ability to discriminate inflammatory from irritable bowel syndrome. Clin Chem Lab Med. 2008;46: 1275-1280.

2. Donowitz M, Kokke FT, Saidi R. Evaluation of patients with chronic diarrhea. $N$ Engl J Med. 1995;332:725-729.

3. Fine KD, Schiller LR. AGA technical review on the evaluation and management of chronic diarrhea. Gastroenterology. 1999; 116:1464-1486.

4. Starmans R, Fijten GH, Knottnerus JA. One year prognosis of abdominal complaints in general practice: a prospective study of patients in whom no organic cause is found. Br J Gen Pract. 1996;46:715-719.

5. Tibble J, Sigthorsson G, Foster R, et al. Faecal calprotectin and faecal occult blood tests in the diagnosis of colorectal carcinoma and adenoma. Gut. 2001;49:402-408.

6. Poullis A, Foster R, Northfield TC, et al. Review article: faecal markers in the assessment of activity in inflammatory bowel disease. Aliment Pharmacol Ther. 2002;16:675-681.

7. Poullis A, Foster R, Mendall MA, et al. Emerging role of calprotectin in gastroenterology. $J$ Gastroenterol Hepatol. 2003;18:756-762.

8. Voganatsi A, Panyutich A, Miyasaki KT, et al. Mechanism of extracellular release of human neutrophil calprotectin complex. J Leukoc Biol. 2001;70:130-134.

9. D'Incà R, Dal Pont E, Di Leo V, et al. Calprotectin and lactoferrin in the assessment of intestinal inflammation and organic disease. Int J Colorectal Dis. 2007;22:429-437.

10. Roseth AG, Fagerhol MK, Aadland E, et al. Assessment of the neutrophil dominating protein calprotectin in feces. A methodologic study. Scand J Gastroenterol. 1992;27:793-798.

11. Thompson WG, Longstreth GF, Drossman DA, et al. Functional bowel disorders and functional abdominal pain. Gut. 1999;45(suppl II):II43-II47.
12. Longstreth GF, Thompson WG, Chey WD, et al. Functional bowel disorders. Gastroenterology. 2006;130:1480-1491.

13. Drossman DA. The functional gastrointestinal disorders and the Rome III process. Gastroenterology. 2006;130:1377-1390.

14. Camilleri M, Choi MG. Review article: irritable bowel syndrome. Alimen Pharmacol Ther. 1997;11:3-15.

15. Gabay C, Kushner I. Acute phase proteins and other systemic responses to inflammation. New Engl J Med. 1999;340: 448-454.

16. Suffredini AF, Fantuzzi G, Badolato R, et al. New insights into the biology of the acute phase response. J Clin Immunol. 1999;19:203-214.

17. Carroccio A, Iacono G, Cottone M, et al. Diagnostic accuracy of fecal calprotectin assay in distinguishing organic causes of chronic diarrhea from irritable bowel syndrome: a prospective study in adults and children. Clin Chem. 2003;49:861-867.

18. Costa F, Mumolo MG, Bellini M, et al. Role of faecal calprotectin as non-invasive marker of intestinal inflammation. Dig Liver Dis. 2003;35:642-647.

19. Tibble JA, Sigthorsson G, Foster R, et al. Use of surrogate markers of inflammation and Rome criteria to distinguish organic from nonorganic intestinal disease. Gastroenterology. 2002;123:450-460.

20. Kapel N. Faecal inflammatory markers in nutrition and digestive diseases in children. Arch Pediatr. 2004;11:403-405.

21. Steinbakk M, Naess-Andresen CF, Lingaas E, et al. Antimicrobial actions of calcium binding leucocyte L1 protein, calprotectin. Lancet. 1990;336:763-765.

22. Roseth AG. Determination of faecal calprotectin, a novel marker of organic gastrointestinal disorders. Dig Liver Dis. 2003;35:607-609.

23. Meucci G, D'Incà R, Maieron R, et al. Diagnostic value of faecal calprotectin in unselected outpatients referred for colonoscopy: A multicenter prospective study. Dig Liver Dis. 2010;42:191-195.

24. Limburg PJ, Ahlquist DA, Sandborn WJ, et al. Fecal calprotectin levels predict colorectal inflammation among patients with chronic diarrhea referred for colonoscopy. Am J Gastroenterol. 2000;95:2831-2837.

25. Husebye E, Ton H, Johne B. Biological variability of fecal calprotectin in patients referred for colonoscopy without colonic inflammation or neoplasm. Am J Gastroenterol. 2001; 96:2683-2687.

26. Grohmann GS, Glass RI, Pereira HG, et al. Enteric viruses and diarrhea in HIV-infected patients. New Engl J Med. 1993;329:14-20.

27. Wildt S, Nordgaard-Lassen I, Bendtsen F, et al. Metabolic and inflammatory faecal markers in collagenous colitis. Eur $J$ Gastroenterol Hepatol. 2007;19:567-574.

28. Jones J, Boorman J, Cann P, et al. British Society of Gastroenterology guidelines for the management of the irritable bowel syndrome. Gut. 2000;47(suppl 2):ii1-19. 